\title{
ERRATUM (v. 9, n. 5, 1998, p. 488)
}

In the article Spectroscopic Study of the Interaction of Nd3 + with the Amino Acids: Phenomenological 4f-4f Intensity Parameters by S. Jericó, C. R. Carubelli, A. M.G. Massabni, E. B. Stucchi, S. R. de A. Leite and O.
Malta, published in the Journal of the Brazilian Chemical Society 9, 5, 1998, pp. 487-493, p.488 (Table 1) the covalent structures of the ligands are not show in. The correct Table 1 is presented here.

Table 1. Covalent structures of the ligands.

\begin{tabular}{|c|c|c|c|c|}
\hline Name & Structural formula $^{(a)}$ & $\underset{(\alpha-\mathrm{COOH})}{\mathrm{pK}_{1}^{(\mathrm{b})}}$ & $\mathrm{pK}_{2}$ & $\begin{array}{c}\mathrm{pK}_{\mathrm{R}} \\
\text { (side chain) }\end{array}$ \\
\hline Glycine & $\begin{array}{c}\mathrm{COO} \\
\mathrm{I}-\mathrm{C}-\mathrm{H} \\
\mathrm{NH}_{3}^{+}\end{array}$ & 2,34 & $\begin{array}{c}9,60 \\
\left(\alpha-\mathrm{NH}_{3}+\right)\end{array}$ & \\
\hline Aspartic acid & $\underset{\mathrm{NH}_{3}^{+}}{\mathrm{COO}}$ & 1,88 & $\begin{array}{c}9,60 \\
\left(\alpha-\mathrm{NH}_{3}+\right)\end{array}$ & $\begin{array}{c}3,65 \\
(\beta-\mathrm{COOH})\end{array}$ \\
\hline Glutamic acid & $\underset{\mathrm{NH}_{3}^{+}}{\mathrm{H}-\mathrm{COO}}-\mathrm{CH}_{2}^{-}-\mathrm{CH}_{2}-\mathrm{COO}^{-}$ & 2,19 & $\begin{array}{c}9,67 \\
\left(\alpha-\mathrm{NH}_{3}+\right)\end{array}$ & $\begin{array}{c}4,25 \\
(\gamma-\mathrm{COOH})\end{array}$ \\
\hline Histidine & $\begin{array}{c}\mathrm{H}-\mathrm{C}-\mathrm{CH}_{2}- \\
\mathrm{N}_{\mathrm{NH}_{3}{ }^{+}} \\
\mathrm{COO}\end{array}$ & 1,82 & $\begin{array}{c}9,17 \\
\left(\alpha-\mathrm{NH}_{3}+\right)\end{array}$ & $\begin{array}{c}6,00 \\
\text { (imidazole) }\end{array}$ \\
\hline Malic acid & $\mathrm{OH}_{\mathrm{H}}$ & $\begin{array}{c}3,40 \\
(\alpha-\mathrm{COOH})\end{array}$ & - & $\begin{array}{c}5,11 \\
(\beta-\mathrm{COOH})\end{array}$ \\
\hline Aspartame & (3) & $2,40^{\text {(c) }}$ & - & - \\
\hline
\end{tabular}

(a) Ionic forms predominating at $\mathrm{pH} 7,0$; (b) The pKa values from the CRC Handbook of Chemistry and Physics ${ }^{21}$; (c) Estimated value from titration curves in this work. 